\title{
28 Research Square \\ Echocardiographic Characteristics and Prognosis of the Fetuses with Ebstein Anomaly in Pregnancy
}

\section{Yuduo Wu}

Capital Medical University Affiliated Anzhen Hospital

Xiaoyan Gu

Capital Medical University Affiliated Anzhen Hospital

Hongjia Zhang

Capital Medical University Affiliated Anzhen Hospital

Yihua He ( $\nabla$ yihuaheecho@163.com )

Capital Medical University Affiliated Anzhen Hospital

Research article

Keywords: Ebstein anomaly, fetal, echocardiography, prognosis

Posted Date: November 2nd, 2020

DOI: https://doi.org/10.21203/rs.3.rs-99088/v1

License: (c) (i) This work is licensed under a Creative Commons Attribution 4.0 International License.

Read Full License 


\section{Abstract}

Objective: This study aimed to describe the fetal echocardiographic features of Ebstein anomaly $(E A)$ and evaluate the prognosis of those fetuses.

Methods: We retrospectively reviewed the clinical records of 53 fetuses with EA. They were enrolled from January 2011 to December 2018 in Beijing Anzhen Hospital and analyzed the fetuses' ultrasonic features and prognosis.

Results: In this single-institutional study, we screened 53 fetuses during 23 39 weeks of gestation with EA. In these fetuses, patients with complications accounted for a more significant percentage $(69.81 \%)$. Ultrasound imaging showed that the posterior lobe shifted down further than the septal leaflets, and the anterior lobe changed less than $50 \%$. Severe tricuspid valve (TV) regurgitation showed the highest

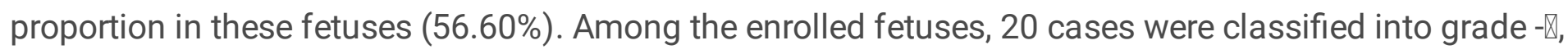
40 cases $(75.47 \%)$ were inducted, and 13 cases (24.5\%) were continued pregnancy. However, only one of those fetuses successful delivery.

Conclusion: Echocardiography has become an essential diagnostic tool in identifying fetuses with EA. The prognosis of the fetuses with EA is relatively low in our center. Therefore, understanding the echocardiographic characteristics and the prognosis of the fetuses with EA is of great value for pregnancy consultations during the perinatal period.

\section{Introduction}

Ebstein anomaly (EA) is a congenital disability of the tricuspid valve (TV), characterized by varying degrees of downward displacement of the TV leaflets into the cavity of the right ventricle ${ }^{1}$. EA accounts for less than $1 \%$ of all cases of congenital heart disease ${ }^{2}$. Current reports predominantly describe the postnatal diagnosis of EA in neonates or infants. However, the EA's fetal presentation is predicted to have a poor overall prognosis as the presentation's age depends on the severity of anatomic and hemodynamic derangements ${ }^{3}$.

In this study, we evaluated the echocardiographic features and the fetus's prognosis with EA in pregnancy. Additionally, we analyzed the echocardiographic data that can be used as potential prognostic factors for fetuses with EA and tricuspid valve dysplasia to provide more valuable information on perinatal consultation.

\section{Materials And Method}

\section{Patient selection}

34,756 fetuses were examined from January 2011 to December 2018 in Beijing Anzhen Hospital, Capital Medical University, Beijing, China. We retrospectively collected 53 fetuses (all singletons) who were 
diagnosed with EA in the tertiary centers' databases, including prenatal demographic, clinical, and echocardiographic data. All procedures involving human participants followed to the national research committee's ethical standards, and the Helsinki declaration and its later amendments or comparable ethical standards.

\section{Data collection}

A complete fetal echocardiographic examination, including two-dimensional (2D), M-mode, color, and pulse Doppler echocardiography, was performed according to the guidelines of the American Society of Echocardiography ${ }^{4}$ to ascertain the diagnosis of EA. Gestational age was confirmed by both the last menstrual period of the mother and early- pregnancy sonographic biometric parameters. A systematic fetal echocardiographic examination was performed, right atria (RA), left atria (LA), atrialized right ventricular (aRV), functional right ventricular (fRV), and left ventricular (LV) were measured separately. In 25 cases, the area ratio of $(R A+a R V) /(L A+L V+f R V)$ was calculated (Fig. 1). According to these data, We divided the tricuspid valve downward shift of the fetuses into four levels, which the severity is associated with the prognosis of EA patients. The classification standard set as follows: Grade I $<0.5$, excellent prognosis; Grade II 0.5-0.99, good prognosis, the survival rate is $92 \%$; Grade III 1-1.49, poor prognosis, the early mortality rate is $10 \%$; Grade IV $\geq 1.5$, inferior prognosis, and the mortality rate is $100 \%$.

\section{Equipment}

All ultrasound examinations were performed by experienced operators using the General Electric Voluson E8 ultrasound system with transabdominal 2- to 4- MHz curvilinear transducers (GE Healthcare Ultrasound, Milwaukee, WI, USA) or the Aloka SSD ultrasound system (Aloka, Tokyo, Japan) using transabdominal 3- to 6- $\mathrm{MHz}$ curvilinear transducers.

\section{Statistical analyses}

Data were analyzed using the Statistical Package for Social Sciences version 22.0 (IBM, Armonk, NY, USA). Jarque-Bera test was used to test variables distribution. Continuous variables are presented as mean \pm standard deviation (SD).

\section{Results}

We evaluated the outcomes of 53 pregnant patients with EA. The pregnant women were aged from 17 to 40 , with an average of 28 . All the women enrolled had a single pregnancy. At the time of initial diagnosis, the gestational age ranged from 23 to 39 weeks, with an average of $28^{+6}$ weeks.

\section{Fetal echocardiographic characteristics}

The abnormalities associated with EA seen on fetal echocardiograms included displacement of the septal and posterior leaflets of the tricuspid valve, lesions of the anterior leaflet, tricuspid regurgitation, and associated deformities. The echocardiographic findings are listed in Table 1 and Fig. 1. 
Table 1

Echocardiographic characteristics of fetuses with EA

\begin{tabular}{|c|c|}
\hline \multicolumn{2}{|l|}{ Item } \\
\hline Pregnant age (years) & $17-40$ \\
\hline Gestational age at admission (weeks) & $23-39$ \\
\hline \multicolumn{2}{|l|}{ Fetuses with comorbidity $\mathrm{n}(\%)$} \\
\hline Yes & $37(69.81 \%)$ \\
\hline No & $16(30.19 \%)$ \\
\hline \multicolumn{2}{|l|}{ The downward displacement of the TV } \\
\hline Septal leaflets $₫ \mathrm{~mm} \rrbracket$ & $6.86 \pm 3.40$ \\
\hline Posterior leaflets $₫ \mathrm{~mm} \rrbracket$ & $9.11 \pm 4.76$ \\
\hline Changed in anterior leaflets $\mathrm{n}(\%)$ & $19 \rrbracket 35.85 \% \rrbracket$ \\
\hline \multicolumn{2}{|l|}{ TV regurgitation } \\
\hline Mild n (\%) & $6 \otimes 11.32 \% \rrbracket$ \\
\hline Moderate n (\%) & $15 \rrbracket 28.30 \% \rrbracket$ \\
\hline Severe n (\%) & $32 \bigotimes 56.60 \% \rrbracket$ \\
\hline Severely n (\%) & $2 \varangle 3.77 \% \rrbracket$ \\
\hline \multicolumn{2}{|l|}{ Area ratio class at gestationn (\%) } \\
\hline I & $1 \rrbracket 4.00 \% \rrbracket$ \\
\hline II & $2 \varangle 8.00 \% \rrbracket$ \\
\hline III & $20 \rrbracket 80.00 \% \rrbracket$ \\
\hline IV & $2 \varangle 8.00 \% \bigotimes$ \\
\hline$T V=$ tricuspidvalve; Area ratio $=(R A+a R V$ & $(L A+L V+f R V)$ \\
\hline
\end{tabular}

\section{Fetal and postnatal mortality and morbidity}

In this study, 17 cases (\%) of all enrolled fetuses were continued pregnancy. Among the 53 fetuses, 16 cases showed simple EA, but 37 cases showed EA-associated malformations (Fig. 2). The pregnant women who continued with the pregnancy just delivered one of the fetuses successfully. In this study, the fetus's birth rate was $1.89 \%(1 / 53)$, the prenatal echocardiogram for this fetus showing in Fig. 3 . We obtained the first postnatal echocardiography on the second day after birth, showing us that tricuspid 
septal displacement deformity, moderate tricuspid regurgitation, and a slightly dilated LA (Fig. 4). The newborn had no other symptoms of discomfort and conducted regular reexamination.

We generated the distribution histogram according to the number of pregnant women with distinct fetal malformations (Fig. 5). The number of pregnant women who chose to terminate their pregnancy is relatively high, 40 in total (in red), including 10 without complications and 30 with complications. In contrast, the number of pregnant women who chose to continue their pregnancy is relatively low, 13 in total (in blue), including 6 without complications and 7 with complications.

\section{Discussion}

Ebstein's anomaly (EA) is a rare congenital heart disorder. Fetal diagnosis and presentations of the disease are typically the most severe and are associated with the highest mortality rates ${ }^{5,6}$. In the present study, we found that fetal echocardiography is a preferred tool to accurately diagnose the lesion. Generally, we can quickly make an accurate judgment on the disease on the four-chamber view for the disease. The images mainly showed that the tricuspid septal and posterior lobe could shift down, causing severe dysplasia. The anterior lobe is attached to the annulus' average level, which may be larger or sailshaped $^{6}$.

Nonetheless, each patient has its characteristics, mainly manifested in the differences among the tricuspid septal lobe, posterior lobe lesions, and occasionally anterior lobular abnormalities that shift down or block the right ventricular outflow tract. Additionally, the right ventricle looks abnormal, the right ventricle cavity below the tricuspid valve significantly reduced, and the trabecular part also becomes smaller $^{7-8}$. The narrowed funnel part includes residual valve tissue or abnormal muscle bundles or fiber bundles8. The 53 cases of EA fetuses in this study were all diagnosed by echocardiography, and each fetal's pathology has its characteristics.

The diagnose of EA can prenatally be granted the prenatal ultrasound examination reveals the above manifestations of the fetal heart. Since this complex congenital anomaly has a very variable anatomical and clinical spectrum, early diagnosis can provide patients with a timely and effective perinatal treatment plan. These outcomes are consistent with previous studies describing ${ }^{9,10,11}$ the high detection rate of other congenital complex diseases with prenatal ultrasound, indicating that echocardiography is worth pursuing in clinical practice.

Fetuses diagnosed with EA have a poor prognosis, and the total mortality rate is as high as $80 \%$ or more $^{12}$. Mild EA had a better prognosis compared to severe EA. However, when combined EA with other diseases, the prognosis is worse, consistent with our study's conclusions. Other studies also reported that factors, including fetal edema, cardiac hypertrophy, outflow obstruction, and cardiothoracic ratio $>0.55$, suggest poor prognosis for the fetuses ${ }^{13}$. Upon longer duration of TV malformation, the disease will be getting severe gradually; the right heart will be overloaded, thus inducing right heart failure and increasing the burden of the right heart. 
In severe cases, the right heart can be twice the size as the left heart, indicating that the fetus's prognosis is inferior and requires timely treatment to ensure the fetal's safety. Issues regarding the importance of the atrialized chamber have also been raised ${ }^{14-15}$. In this study, only one fetus was regularly followed up and examined by our center with an induction rate of $98.11 \%(1 / 53)$, which is much higher than EA patients' mortality rate in previous studies. The main reason is that fetuses with severe or severely late tricuspid regurgitation and those with combined malformations account for a large proportion of cases.

Our center is a cardiovascular hospital, and most of the patients are mothers with critically ill fetuses. Although we can make the diagnosis in time, the treatment efforts during the perinatal period cannot be exerted, contributing to patients' low birth rate. Such a high induction rate can also explain the high proportion of grade- $\varangle$ and grade- $\varangle$ in prognosis's grading area in our study. On the other hand, it also shows that we can identify more congenital heart diseases in the fetal period with examination assistive technology. It is noteworthy that the high detection rate is also a factor contributing to the increase in the death rate, although this needs further verification.

Although this is a relatively larger cohort of fetuses with EA, the sample size is still relatively small, limiting to providing a firm conclusion. Additionally, this is a single-institutional retrospective study. Many patients were coming to us from other centers. As a result, potential patient selection bias may have led to the data, and the results presented here may not be directly applicable to other centers and populations.

\section{Conclusions}

Through the study of EA fetuses in our center, we found that EA is complex congenital heart disease with a poor prognosis. Although the fetus can be clearly diagnosed during the fetal period, it is still impossible to intervene in the fetus. It is currently a significant factor affecting the prognosis of these children.

Because we are a professional fetal medical center, most of the fetuses are abnormal. They have severe congenital heart disease, which will lead to the inclusion of children with poor prognosis in this study, bringing bias to the study. We hope to intervene in some patients during the fetal period shortly so that they can be born smoothly and have the opportunity to undergo postnatal treatment. At the same time, we also hope that our center can train more excellent sonographers to take root in the grassroots units. Alleviate the unevenness of medical resources in China.

\section{Abbreviations}

EA: Ebstein anomaly; TV: Tricuspid valve; RA: Right atria; LA: Left atria; aRV: Atrialized right ventricule; fRV:functional right ventricule; LV: Left ventricule.

\section{Declarations}

Acknowledgements 
Not applicable.

\section{Authors' contributions}

Yuduo Wu was responsible for data collection, data analysis, and manuscript preparation; Xiaoyan Gu was responsible for data interpretation; Yihua He was responsible for manuscript revision and finalization. Funding was secured by Hongjia Zhang.

\section{Founding}

This study was supported by National Science Funding (No.81301241), Beijing Key Laboratory of Maternal-Fetal Medicine and Fetal Heart Disease (No. BZ0308), and Beijing Municipal Administration of Hospitals' Ascent Plan (No. DFL20180602).

\section{Availability of data and materials}

The datasets analyzed for the current study are available from the correspond- ing author on reasonable request.

\section{Ethics approval and consent to participate}

The human study were approved by the ethics committee of Beijing Anzhen Hospital. Written informed consent was obtained from all subjects.

\section{Consent for publication}

Not applicable.

\section{Competing interests}

The authors declare that they have no competing interests.

\section{References}

1. I G, L G, R S, et al. Ebstein's Anomaly of the Tricuspid Valve in the Fetus- AMulticenter Experience.[J]. Ultraschall in der Medizin (Stuttgart, Germany : 1980), 2017,38(4):427-436.

2. Shetty R K, Vivek G, Nayak K, et al. Fetal Ebstein's anomaly[J]. BMJ Case Rep, 2012,2012.

3. LR F, MC E, BT K, et al. Outcomes and Predictors of Perinatal Mortality in Fetuses With Ebstein Anomaly or Tricuspid Valve Dysplasia in the Current Era: A Multicenter Study.[J]. Circulation, 2015,132(6):481-489.

4. Rychik J, Ayres N, Cuneo B, et al. American Society of Echocardiography guidelines and standards for performance of the fetal echocardiogram. J Am Soc Echocardiogr. 2004;17:803- 
5. Freud L R, Escobar-Diaz M C, Kalish B T, et al. Outcomes and Predictors of Perinatal Mortality in Fetuses With Ebstein Anomaly or Tricuspid Valve Dysplasia in the Current Era: A Multicenter Study[J]. Circulation, 2015,132(6):481-489.

6. Gottschalk I, Gottschalk L, Stressig R, et al. Ebstein's Anomaly of the Tricuspid Valve in the Fetus - A Multicenter Experience[J]. Ultraschall Med, 2017,38(4):427-436.

7. Prota, C. and G. Di Salvo, et al. (2019). "Prognostic value of echocardiographic parameters in pediatric patients with Ebstein's anomaly." Int J Cardiol 278: 76-83.

8. Booker O J, Nanda N C. Echocardiographic assessment of Ebstein'sanomaly[J]. Echocardiography, 2015,32Suppl 2:S177-S188.

9. Gottschalk I, Gottschalk L, Stressig R, et al. Ebstein's Anomaly of the Tricuspid Valve in the FetusAMulticenter Experience[J]. Ultraschall Med, 2017,38(4):427-436.

10. Kaya B, Ekiz A, Acar D K, et al. Prenatal diagnosis of aortopulmonary window by foetal echocardiography: 'U or reversed U sign'[J]. J ObstetGynaecol, 2019,39(5):712-713.

11. Yu S, Han J, Gao S, et al. The prenatal diagnosis of aortopulmonary window by fetal echocardiography[J]. Echocardiography, 2018,35(11):1835-1840.

12. Garcia C, Alvarez T, Bravo C, et al. Prenatal Diagnosis of an Aortopulmonary Window With an Interrupted Aortic Arch[J]. J Ultrasound Med, 2016,35(10):2087-2093.

13. Pornprasertchai V, Vijarnsorn C, Kanjanauthai S, et al. Contemporary outcomes and mortality risks of Ebstein anomaly: A single-center experience in Thailand[J]. Congenit Heart Dis, 2019.

14. Zimmer E Z, Blazer S, Lorber A, et al. Fetal Ebstein's anomaly: early and late appearance[J]. PrenatDiagn, 2012,32(3):228-233.

15. RS L, LG A, MA A, et al. Diagnosis and prognosis of fetal ebstein anomaly.[J]. Ginecologia y obstetricia de Mexico, 2014,82(2):93-104.

16. Selamet T E, McElhinney D B, Freud L R, et al. Assessment of Progressive Pathophysiology After Early Prenatal Diagnosis of the Ebstein Anomaly or Tricuspid Valve Dysplasia[J]. Am J Cardiol, 2017,119(1):106-111.

\section{Figures}




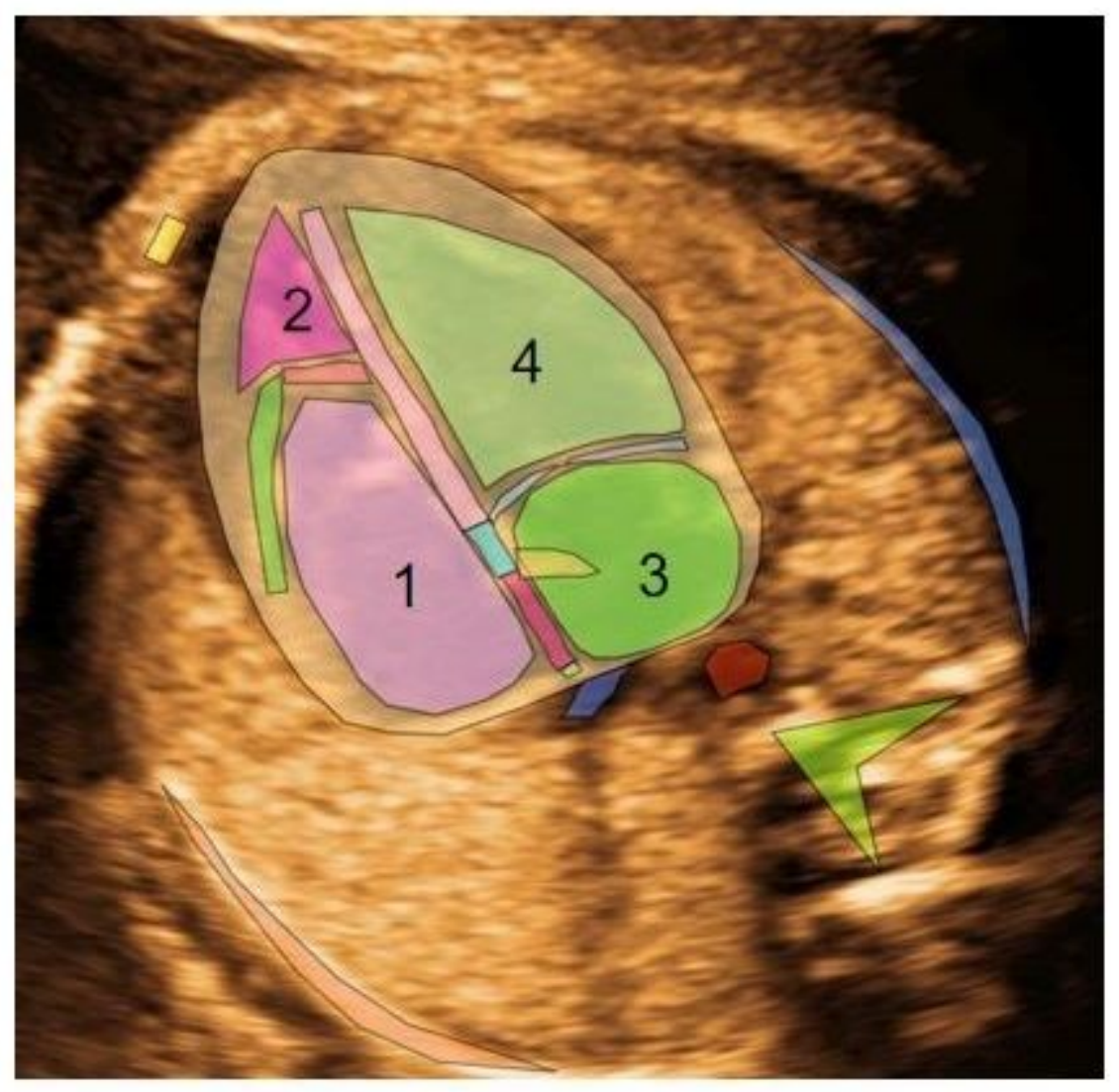

\section{Figure 1}

Measurements were done in a four-chamber section of the heart at the end of diastole. Area ratio $=(\mathrm{RA}+$ $\mathrm{aRV}) /(\mathrm{fRV}+\mathrm{LA}+\mathrm{LV}) .1=\mathrm{RA}+\mathrm{aRV}, 2=\mathrm{fRV}, 3=\mathrm{LA}, 4=\mathrm{LV}$. Among the enrolled fetuses, twenty-five cases were

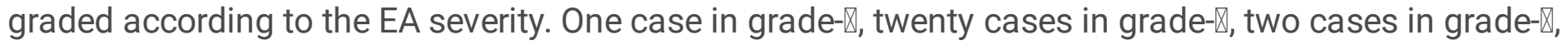

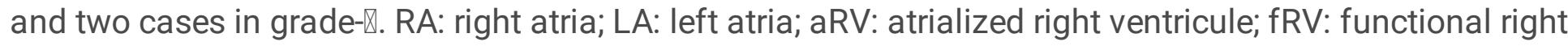
ventricule; LV: left ventricule. 


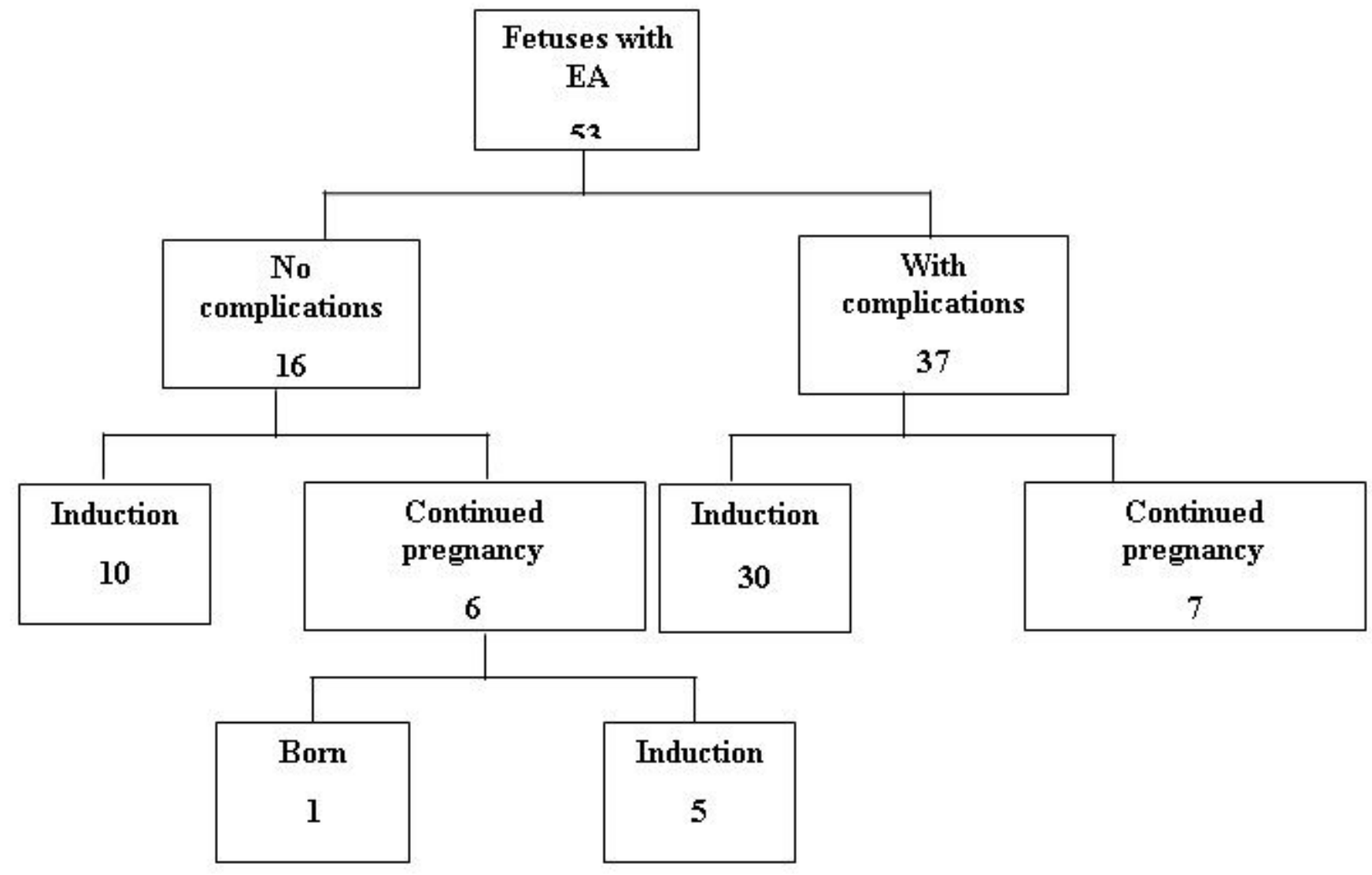

Figure 2

A schema of echocardiographic analysis for the enrolled fetuses with EA.
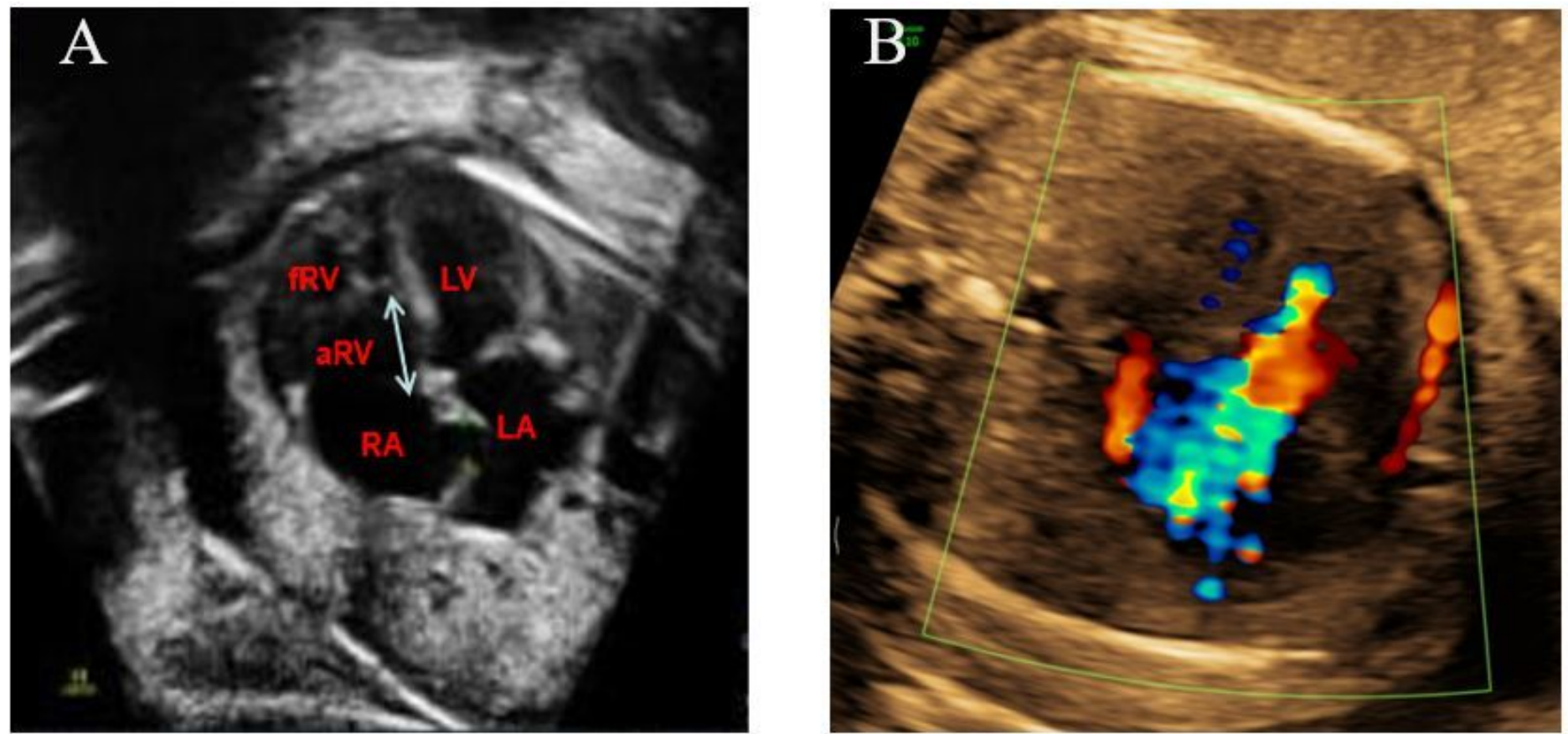

Figure 3 
Prenatal ultrasound examinations for the developing fetus in a four-chamber view. (A) Two-dimensional ultrasound. Two-way arrows indicate the downward shift of septum tricuspid at 28 wks of gestation. (B) Color flow mapping. Color Doppler flow imaging indicates a moderate tricuspid regurgitation before birth (the arrow mark). tRA: right atrium, aRV: Atrialized right ventricle, fRV: Functional right ventricle, LA: left atrium; LV: left ventricle.
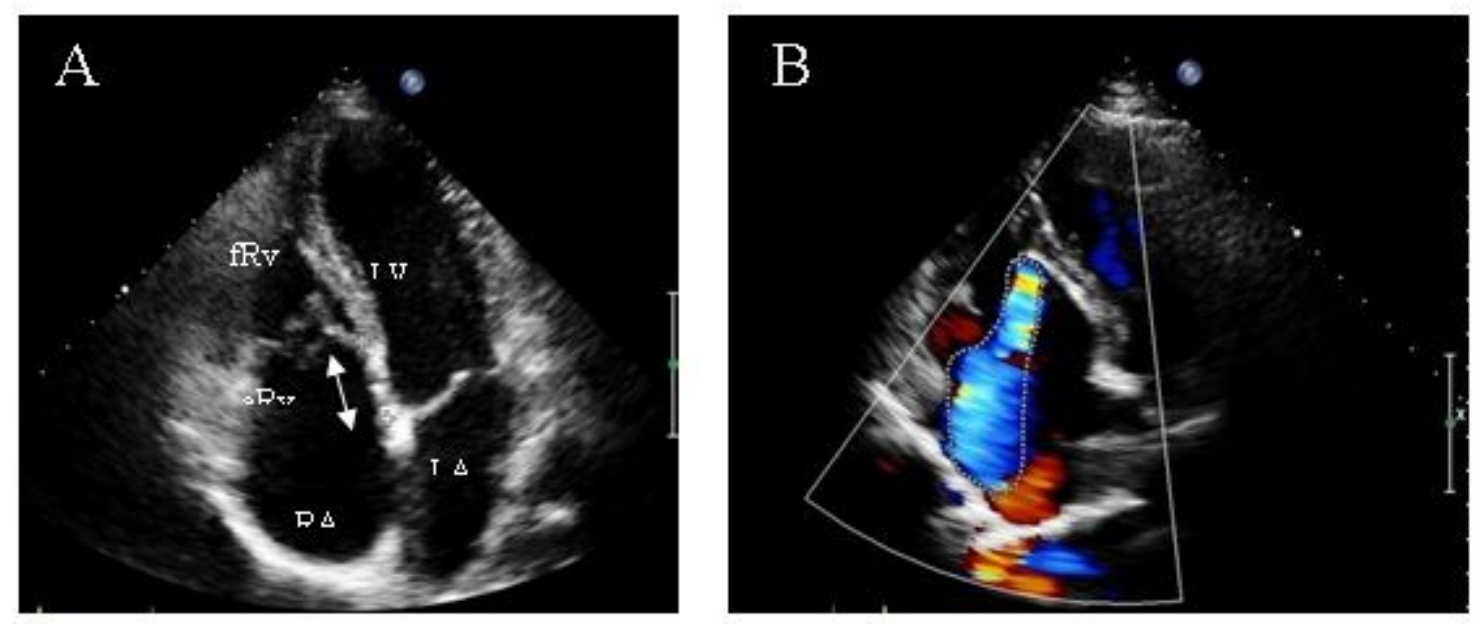

\section{Figure 4}

The postnatal ultrasound examination from the developing fetus in the four-chamber view. (A) Twodimensional ultrasound. Two-way arrows indicate the TV septal descending. (B) Color flow mapping. Color Doppler indicates the tricuspid regurgitation. TV=tricuspid valve.
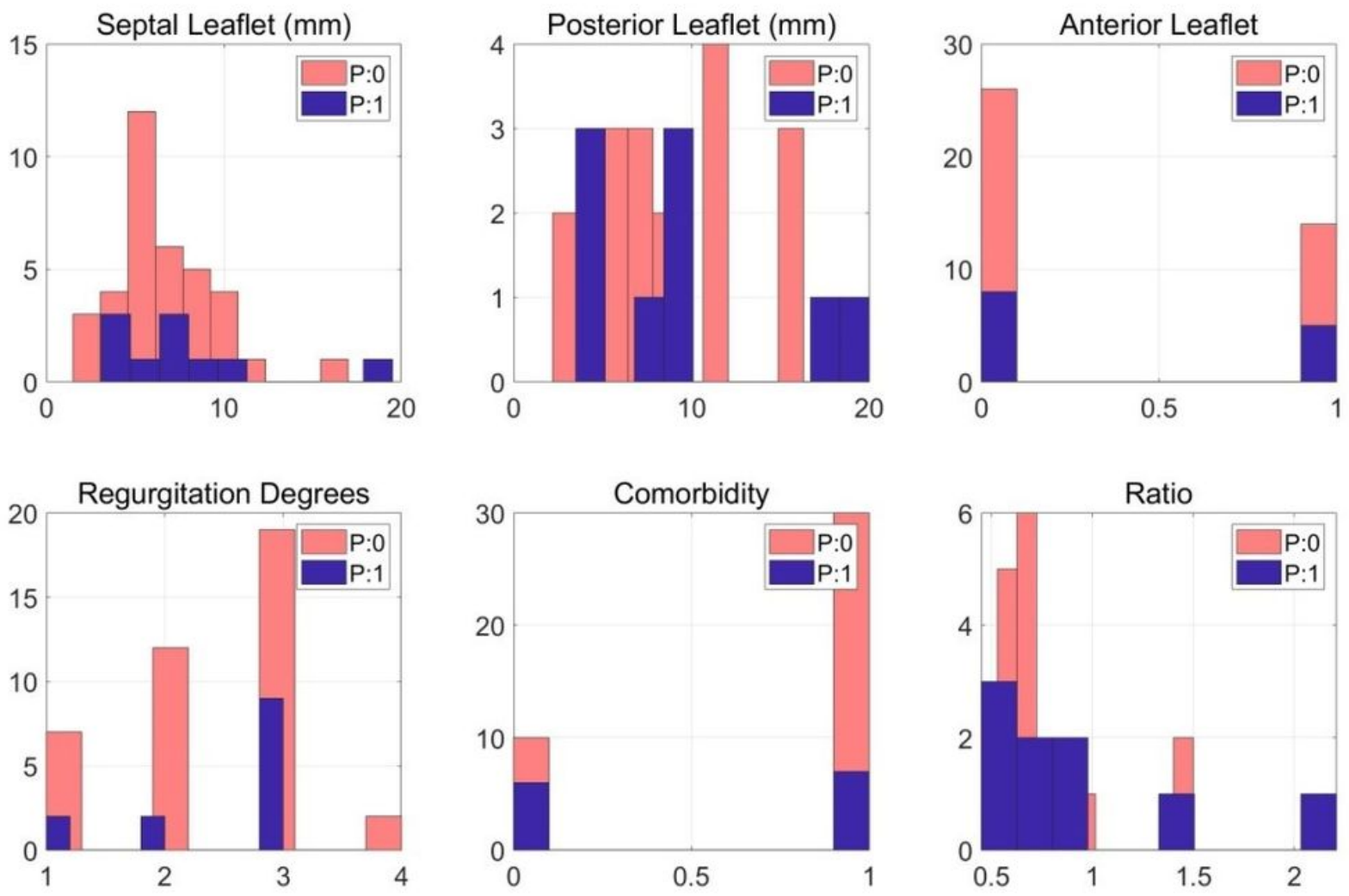


\section{Figure 5}

The distribution of patients was presented under each relevant indicator. $\mathrm{P}=0$ termination of pregnancy (red), $P=1$ continuation of pregnancy (blue). 